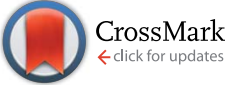

Cite this: RSC Adv., 2017, 7, 15997
Received 22nd January 2017 Accepted 27th February 2017

DOI: $10.1039 / \mathrm{c} 7 \mathrm{ra00954b}$

rsc.li/rsc-advances

\section{Semisynthesis of some matrine ether derivatives as insecticidal agents $\uparrow$}

\begin{abstract}
Jiu-Lin Huang, $\$$ Min LV $\$$ and Hui Xu*
In continuation of our program to discover new potential natural-product-based crop protection agents, we synthesized a series of 14-formyl-15-aryloxy/methoxymatrine and 14-aryloxymethylidenylmatrine derivatives as pesticidal agents by structural modification of matrine, a biorenewable quinolizidine alkaloid isolated from Sophora flavescens. The structural assignment was based on spectroscopic and X-ray analysis data. Their pesticidal activities were carried out against two typically crop-threatening agricultural insect pests, Mythimna separata Walker and Plutella xylostella Linnaeus. Compounds $4 \mathrm{i}$ and $4 \mathrm{k}$ exhibited more potent oral toxicity than matrine against 3rd-instar larvae of $P$. xylostella. As compared with matrine, all derivatives displayed a growth inhibitory property against early 3rd-instar larvae of $M$. separata, and in particular compounds $4 \mathbf{i}-\mathbf{k}$ displayed more promising insecticidal activity than toosendanin. Some interesting results of structure-activity relationships were also observed.
\end{abstract}

\section{Introduction}

Mythimna separata Walker (oriental armyworm) and Plutella xylostella Linnaeus (diamondback moth) are two widely distributed and serious typical lepidopteran insect pests. Their infestations are very troublesome and hard to control. ${ }^{1,2}$ In 2012, intermittent outbreaks of $M$. separata occurred widely in China, and about 4 million hectares of crops were completely lost. ${ }^{3}$ Although lots of chemical pesticides have been extensively applied to deal with insect pest outbreaks, insect pest resistance and negative impacts on human health and environmental safety have emerged. ${ }^{4-7}$ Therefore, the development of new potential alternatives to effectively and selectively control insect pests is highly urgent.

Matrine (1, Fig. 1) is a biorenewable quinolizidine alkaloid isolated from the roots of Sophora flavescens (Kushen), which is widely distributed in Asia, Oceanica, and the Pacific islands. ${ }^{8,9}$ Matrine and its derivatives exhibited a broad scope of biological properties such as anticancer, anti-inflammatory, and antiviral activities. ${ }^{10-16}$ Meanwhile, compound 1 also exhibited potent insecticidal activity in the agricultural field. ${ }^{17-19}$ On the other hand, it was found that the introduction of an acrylic aldehyde scaffold (fragment A, Fig. 1) into dehydroepiandrosterone could produce derivatives $\mathbf{I}^{-\mathbf{I V}^{\mathbf{2 0}-22}}$ (Fig. 1), which showed potent antiproliferative effects. Moreover, their lipophilicity, which

Research Institute of Pesticidal Design \& Synthesis, College of Chemistry \& Pharmacy/Plant Protection, Northwest A\&F University, Yangling 712100, Shaanxi Province, China. E-mail: orgxuhui@nwsuaf.edu.cn; Fax: +86-29-87091952; Tel: +8629-87091952

$\uparrow$ CCDC 1522361, 1524882, 1524886 and 1524887. For crystallographic data in CIF or other electronic format see DOI: $10.1039 / \mathrm{c} 7 \mathrm{ra00954b}$

\$ These authors contributed equally to this work. improves their ability to cross through cell membranes, was increased, whereas their side effects and toxicity were decreased. ${ }^{21}$ Meanwhile, to the best of our knowledge, little attention has been paid to the structural modifications of compound 1 as pesticidal agents. Based upon the above interesting results, to discover biorenewable matrine-based pesticides, herein an acrylic aldehyde scaffold was introduced into compound 1, followed by transformation to 14-formyl-15aryloxy/methoxymatrine (4, Fig. 1). Their pesticidal activities were evaluated against two typically crop-threatening insect pests, M. separata and P. xylostella.

\section{Materials and methods}

\section{General}

All chemical reagents were purchased and utilized without further purification. Compound $\mathbf{1}$ was purchased from Baoji Haoxiang Bio-technology Co. Ltd. Melting point (mp) was determined using the XT- 4 digital mp apparatus. Optical rotation was measured using an Autopol III automatic polarimeter. Infrared (IR) spectra were measured by a TENSOR 27 spectrometer. Proton nuclear magnetic resonance spectra $\left({ }^{1} \mathrm{H}\right.$ NMR) were measured with Avance III $500 \mathrm{MHz}$ equipment. Highresolution mass spectra (HRMS) were carried out with an LTQ FT Ultra instrument. Microwave irradiation was performed in a CEM Discover Synthesis Unit.

\section{Synthesis of 14-formyl-15-chloromatrine (2)}

$\mathrm{POCl}_{3}(15 \mathrm{mmol})$ was added slowly to a solution of DMF (1.2 $\mathrm{mL})$ in dry $\mathrm{CH}_{2} \mathrm{Cl}_{2}(5 \mathrm{~mL})$ at $0^{\circ} \mathrm{C}$. After addition, the mixture was stirred for $1 \mathrm{~h}$ at $0{ }^{\circ} \mathrm{C}$. Then, a solution of $1(5 \mathrm{mmol})$ in dry $\mathrm{CH}_{2} \mathrm{Cl}_{2}(15 \mathrm{~mL})$ was added dropwise to the above mixture. After 


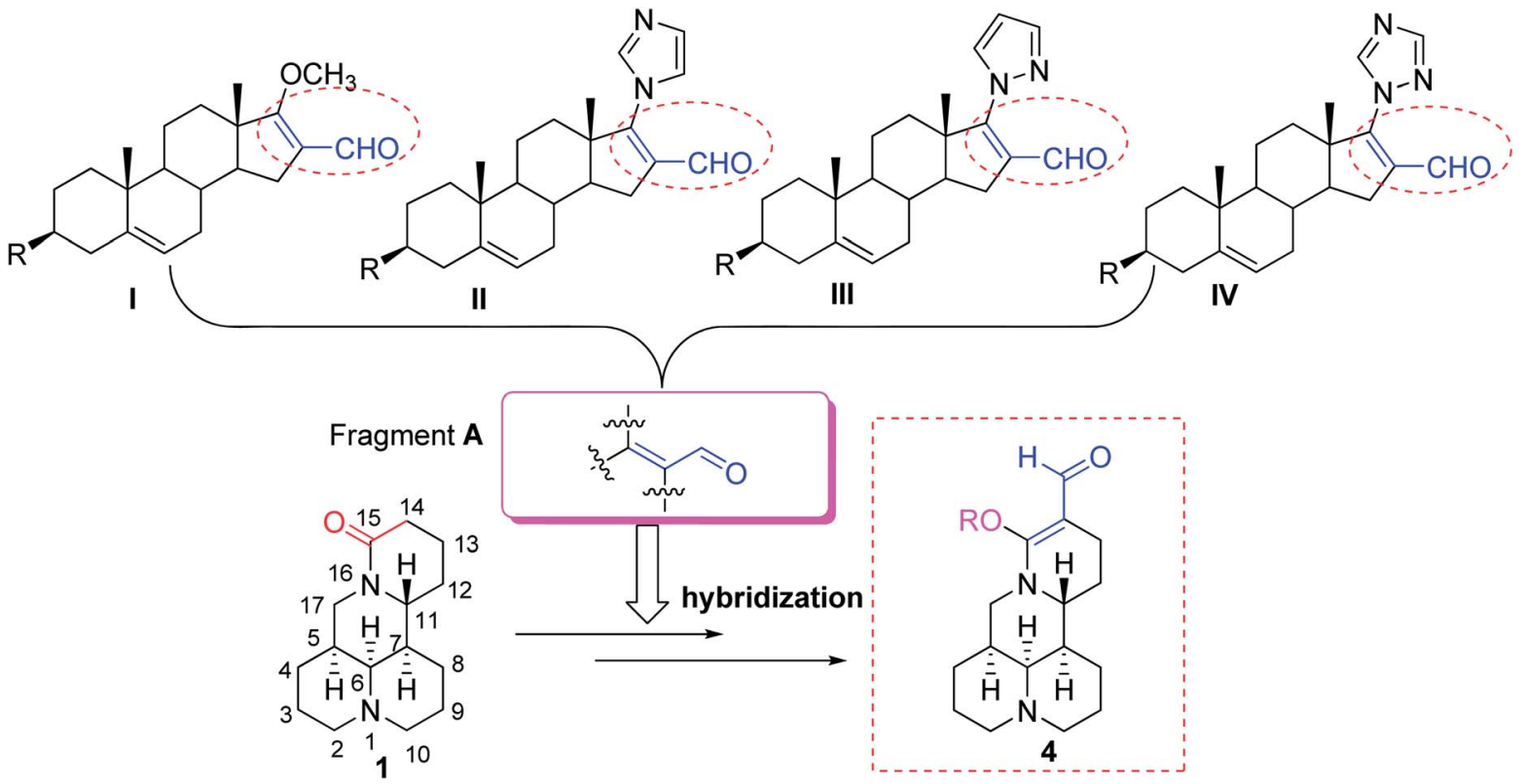

Fig. 1 Chemical structures of matrine (1), its derivatives (4), and other dehydroepiandrosterone derivatives (I-IV).

addition, the reaction temperature was slowly raised to room temperature, and the reaction process was checked by TLC analysis. After $9 \mathrm{~h}$, the mixture was concentrated in vacuo, and 5 $\mathrm{mL}$ of ice water was added. The $\mathrm{pH}$ value of the mixture was adjusted with $40 \%$ aq. $\mathrm{NaOH}$ to $8-9$. Subsequently, hydrolysis of the mixture was conducted at $60{ }^{\circ} \mathrm{C}$ for $2 \mathrm{~h}$. After cooling, the pale yellow precipitate was filtered and washed with water. Finally, the solid was purified by silica gel column chromatography eluted with dichloromethane/methanol to give 2 (92\% yield) as a pale yellow solid.

\section{Data for 2}

Mp 106-108 ${ }^{\circ} \mathrm{C} ;[\alpha]_{\mathrm{D}}^{20}=-83\left(c 2.6 \mathrm{mg} \mathrm{mL}^{-1}, \mathrm{CHCl}_{3}\right) ; \mathrm{IR} \mathrm{cm}^{-1}$ (KBr): 2776, 1635, 1564; ${ }^{1} \mathrm{H}$ NMR $\left(500 \mathrm{MHz}, \mathrm{CDCl}_{3}\right) \delta: 9.67$ (s, $1 \mathrm{H}), 4.06-4.10(\mathrm{~m}, 1 \mathrm{H}), 4.01(\mathrm{dd}, J=13.0,4.0 \mathrm{~Hz}, 1 \mathrm{H}), 3.59(\mathrm{t}, J$ $=13.0 \mathrm{~Hz}, 1 \mathrm{H}), 2.79-2.86(\mathrm{~m}, 2 \mathrm{H}), 2.39-2.44(\mathrm{~m}, 1 \mathrm{H}), 2.27-2.33$ (m, 1H), $2.18(\mathrm{~s}, 1 \mathrm{H}), 1.96-2.03(\mathrm{~m}, 2 \mathrm{H}), 1.80-1.92(\mathrm{~m}, 4 \mathrm{H})$, 1.55-1.71 (m, 5H), 1.43-1.49 (m, 2H), 1.32-1.40 (m, 1H); HRMS (ESI): calcd for $\mathrm{C}_{16} \mathrm{H}_{24} \mathrm{ON}_{2} \mathrm{Cl}\left([\mathrm{M}+\mathrm{H}]^{+}\right), 295.1572$; found, 295.1571.

\section{General procedure for synthesis of $4 a-e, k, 1$}

A mixture of $2(0.3 \mathrm{mmol}), \mathrm{KOH}(0.6 \mathrm{mmol})$, and different phenols (3a-d, $\mathbf{k}, \mathbf{l}, 0.9 \mathrm{mmol})$ in DMF $(5 \mathrm{~mL})$ were stirred at $120{ }^{\circ} \mathrm{C}$ for $2-9 \mathrm{~h}$; or a mixture of $2(0.3 \mathrm{mmol})$ and $\mathrm{KOH}(1.5$ $\mathrm{mmol})$ in $\mathrm{MeOH}(3 \mathbf{e}, 5 \mathrm{~mL})$ was refluxed for $14 \mathrm{~h}$. Then the reaction mixture was diluted with water $(10 \mathrm{~mL})$, and extracted with ethyl acetate $(15 \mathrm{~mL} \times 3)$. Subsequently, the combined organic phase was washed by saturated aq. $\mathrm{Na}_{2} \mathrm{CO}_{3}(20 \mathrm{~mL} \times 2)$, dried over anhydrous $\mathrm{Na}_{2} \mathrm{SO}_{4}$, concentrated in vacuo, and purified by PTLC eluted with dichloromethane/methanol to afford target compounds $\mathbf{4 a - e , ~} \mathbf{k}, \mathbf{l}$.

\section{General procedure for synthesis of $\mathbf{4 f}-\mathbf{j}$}

Compound $2(0.3 \mathrm{mmol})$ reacted with different phenols ( $\mathbf{3 f}-\mathbf{j}$, $0.9 \mathrm{mmol})$ in the presence of $\mathrm{K}_{2} \mathrm{CO}_{3}(0.66 \mathrm{mmol})$ and DMF $(0.5 \mathrm{~mL})$ under microwave irradiation at $120^{\circ} \mathrm{C}$ for $20 \mathrm{~min}$. After cooling to room temperature, the reaction mixture was diluted with $\mathrm{CH}_{2} \mathrm{Cl}_{2}(10 \mathrm{~mL})$, and washed by saturated aq. $\mathrm{Na}_{2} \mathrm{CO}_{3}(5 \mathrm{~mL} \times 2)$, dried over anhydrous $\mathrm{Na}_{2} \mathrm{SO}_{4}$, concentrated in vacuo, and purified by PTLC eluted with dichloromethane/methanol to afford target compounds $\mathbf{4} \mathbf{f}-\mathbf{j}$.

\section{Data for 4a}

Yield: $65 \%$, pale yellow solid, $\mathrm{mp} 97-99^{\circ} \mathrm{C} ;[\alpha]_{\mathrm{D}}^{20}=-16($ c $3.3 \mathrm{mg}$ $\mathrm{mL}^{-1}, \mathrm{CHCl}_{3}$ ); IR cm ${ }^{-1}$ (KBr): 3051, 2759, 1635, 1576; ${ }^{1} \mathrm{H}$ NMR $\left(500 \mathrm{MHz}, \mathrm{CDCl}_{3}\right) \delta: 9.39(\mathrm{~s}, 1 \mathrm{H}), 7.29-7.32(\mathrm{~m}, 2 \mathrm{H}), 7.04(\mathrm{t}, J=$ $7.5 \mathrm{~Hz}, 1 \mathrm{H}), 7.01(\mathrm{~d}, J=8.0 \mathrm{~Hz}, 2 \mathrm{H}), 3.95-4.00(\mathrm{~m}, 1 \mathrm{H}), 3.47(\mathrm{dd}$, $J=13.0,3.5 \mathrm{~Hz}, 1 \mathrm{H}), 3.32(\mathrm{t}, J=13.0 \mathrm{~Hz}, 1 \mathrm{H}), 2.74-2.84(\mathrm{~m}, 2 \mathrm{H})$, 2.44-2.50 (m, 1H), 2.35-2.41 (m, 1H), $2.11(\mathrm{~s}, 1 \mathrm{H}), 1.89-2.05(\mathrm{~m}$, $4 \mathrm{H}), 1.73-1.79(\mathrm{~m}, 1 \mathrm{H}), 1.55-1.67(\mathrm{~m}, 4 \mathrm{H}), 1.36-1.46(\mathrm{~m}, 5 \mathrm{H})$; HRMS (ESI): calcd for $\mathrm{C}_{22} \mathrm{H}_{29} \mathrm{O}_{2} \mathrm{~N}_{2}\left([\mathrm{M}+\mathrm{H}]^{+}\right), 353.2224$; found, 353.2221 .

\section{Data for $4 b$}

Yield: $77 \%$, pale yellow solid, mp $134-136{ }^{\circ} \mathrm{C} ;[\alpha]_{\mathrm{D}}^{20}=-18(c$ $3.2 \mathrm{mg} \mathrm{mL}{ }^{-1}, \mathrm{CHCl}_{3}$ ); IR cm ${ }^{-1}(\mathrm{KBr}): 3052,2757,1637,1564$, 1577,$1505 ;{ }^{1} \mathrm{H}$ NMR $\left(500 \mathrm{MHz}, \mathrm{CDCl}_{3}\right) \delta: 9.33(\mathrm{~s}, 1 \mathrm{H}), 7.18(\mathrm{~d}, J=$ $7.0 \mathrm{~Hz}, 1 \mathrm{H}), 7.08(\mathrm{t}, J=7.5 \mathrm{~Hz}, 1 \mathrm{H}), 6.95(\mathrm{t}, J=7.5 \mathrm{~Hz}, 1 \mathrm{H}), 6.84$ (d, $J=8.0 \mathrm{~Hz}, 1 \mathrm{H}), 3.97-3.99(\mathrm{~m}, 1 \mathrm{H}), 3.34(\mathrm{~d}, J=8.0 \mathrm{~Hz}, 2 \mathrm{H})$, 2.74-2.84 (m, 2H), 2.41-2.45 (m, 2H), $2.34(\mathrm{~s}, 3 \mathrm{H}), 2.12(\mathrm{~s}, 1 \mathrm{H})$, 1.90-2.03 (m, 4H), $1.78(\mathrm{~s}, 1 \mathrm{H}), 1.56-1.70(\mathrm{~m}, 4 \mathrm{H}), 1.37-1.46(\mathrm{~m}$, $5 \mathrm{H})$; HRMS (ESI): calcd for $\mathrm{C}_{23} \mathrm{H}_{31} \mathrm{O}_{2} \mathrm{~N}_{2}\left([\mathrm{M}+\mathrm{H}]^{+}\right), 367.2380$; found, 367.2380. 


\section{Data for $4 c$}

Yield: $64 \%$, pale yellow solid, mp $159-161{ }^{\circ} \mathrm{C} ;[\alpha]_{\mathrm{D}}^{20}=-24(c$ $3.0 \mathrm{mg} \mathrm{mL}{ }^{-1}, \mathrm{CHCl}_{3}$ ); IR cm ${ }^{-1}$ (KBr): 3052, 2762, 1635, 1564, 1505; ${ }^{1} \mathrm{H}$ NMR $\left(500 \mathrm{MHz}, \mathrm{CDCl}_{3}\right) \delta: 9.38$ (s, 1H), 7.29 (d, $J=$ $9.0 \mathrm{~Hz}, 2 \mathrm{H}), 6.92$ (d, $J=8.5 \mathrm{~Hz}, 2 \mathrm{H}), 3.95-4.00(\mathrm{~m}, 1 \mathrm{H}), 3.51$ (dd, $J=13.0,4.5 \mathrm{~Hz}, 1 \mathrm{H}), 3.32(\mathrm{t}, J=13.0 \mathrm{~Hz}, 1 \mathrm{H}), 2.74-2.84(\mathrm{~m}, 2 \mathrm{H})$, 2.35-2.47 (m, 2H), $2.12(\mathrm{~s}, 1 \mathrm{H}), 1.90-2.03(\mathrm{~m}, 4 \mathrm{H}), 1.75-1.80(\mathrm{~m}$, 1H), 1.59-1.69 (m, 4H), 1.37-1.46 (m, 5H), 1.29 (s, 9H); HRMS (ESI): calcd for $\mathrm{C}_{26} \mathrm{H}_{37} \mathrm{O}_{2} \mathrm{~N}_{2}\left([\mathrm{M}+\mathrm{H}]^{+}\right)$, 409.2850; found, 409.2849 .

\section{Data for 4d}

Yield: $52 \%$, pale yellow solid, mp $101-103{ }^{\circ} \mathrm{C} ;[\alpha]_{\mathrm{D}}^{20}=-19(c$ $2.0 \mathrm{mg} \mathrm{mL}{ }^{-1}, \mathrm{CHCl}_{3}$ ); IR cm ${ }^{-1}$ (KBr): 3059, 2764, 1635, 1571, 1485; ${ }^{1} \mathrm{H}$ NMR $\left(500 \mathrm{MHz}, \mathrm{CDCl}_{3}\right) \delta: 9.29(\mathrm{~s}, 1 \mathrm{H}), 7.19(\mathrm{~d}, J=$ $11.0 \mathrm{~Hz}, 2 \mathrm{H}), 6.89$ (d, $J=11.0 \mathrm{~Hz}, \mathrm{ArH}, 2 \mathrm{H}), 3.87-3.93(\mathrm{~m}, 1 \mathrm{H})$, 3.24-3.37 (m, 2H), 2.67-2.78 (m, 2H), 2.27-2.42 (m, 2H), 2.05 (s, 1H), 1.83-1.99 (m, 4H), 1.64-1.72 (m, 2H), 1.49-1.60 (m, $4 \mathrm{H}$ ), 1.33-1.40 (m, 4H); HRMS (ESI): calcd for $\mathrm{C}_{26} \mathrm{H}_{28} \mathrm{O}_{2} \mathrm{~N}_{2} \mathrm{Cl}$ $\left([\mathrm{M}+\mathrm{H}]^{+}\right), 387.1834$; found, 387.1833.

\section{Data for $4 \mathrm{e}$}

Yield: $51 \%$, pale yellow solid, $\mathrm{mp} 84-86^{\circ} \mathrm{C} ;[\alpha]_{\mathrm{D}}^{20}=-40($ c $2.8 \mathrm{mg}$ $\left.\mathrm{mL}^{-1}, \mathrm{CHCl}_{3}\right)$; IR cm ${ }^{-1}$ (KBr): 2757, 1623, $1555 ;{ }^{1} \mathrm{H}$ NMR (500 $\left.\mathrm{MHz} \mathrm{CDCl}_{3}\right) \delta: 9.50(\mathrm{~s}, \mathrm{CHO}, 1 \mathrm{H}), 3.86-3.90(\mathrm{~m}, 1 \mathrm{H}), 3.83(\mathrm{~s}$, $3 \mathrm{H}), 3.57$ (dd, $J=12.5,4.5 \mathrm{~Hz}, 1 \mathrm{H}), 3.38(\mathrm{t}, J=12.5 \mathrm{~Hz}, 1 \mathrm{H})$, 2.78-2.85 (m, 2H), 2.25-2.37 (m, 2H), 2.15 (s, 1H), 1.85-2.01 (m, $4 \mathrm{H}), 1.72-1.75(\mathrm{~m}, 2 \mathrm{H}), 1.56-1.67$ (m, 5H), 1.41-1.48 (m, 3H); HRMS (ESI): calcd for $\mathrm{C}_{17} \mathrm{H}_{27} \mathrm{O}_{2} \mathrm{~N}_{2}\left([\mathrm{M}+\mathrm{H}]^{+}\right), 291.2067$; found, 291.2067.

\section{Data for $4 f$}

Yield: $74 \%$, pale yellow solid, $\mathrm{mp} 100-102{ }^{\circ} \mathrm{C} ;[\alpha]_{\mathrm{D}}^{20}=-30(c$ $\left.2.5 \mathrm{mg} \mathrm{mL}{ }^{-1}, \mathrm{CHCl}_{3}\right) ; \mathrm{IR} \mathrm{cm}^{-1}(\mathrm{KBr}): 3043,2761,1632,1574 ;{ }^{1} \mathrm{H}$ NMR (500 MHz, $\left.\mathrm{CDCl}_{3}\right) \delta: 9.34(\mathrm{~s}, 1 \mathrm{H}), 7.16(\mathrm{t}, J=7.5 \mathrm{~Hz}, 1 \mathrm{H})$, $6.86(\mathrm{~d}, J=7.5 \mathrm{~Hz}, 1 \mathrm{H}), 6.79-6.82(\mathrm{~m}, 2 \mathrm{H}), 3.97-4.01(\mathrm{~m}, 1 \mathrm{H})$, $3.49(\mathrm{dd}, J=13.0,4.0 \mathrm{~Hz}, 1 \mathrm{H}), 3.32(\mathrm{t}, J=12.5 \mathrm{~Hz}, 1 \mathrm{H}), 2.75-2.84$ (m, 2H), 2.38-2.50 (m, 2H), $2.33(\mathrm{~s}, 3 \mathrm{H}), 2.12(\mathrm{~s}, 1 \mathrm{H}), 1.92-2.05$ (m, 4H), 1.74-1.80 (m, 1H), 1.58-1.68 (m, 4H), 1.37-1.45 (m, $5 \mathrm{H})$; HRMS (ESI): calcd for $\mathrm{C}_{23} \mathrm{H}_{31} \mathrm{O}_{2} \mathrm{~N}_{2}\left([\mathrm{M}+\mathrm{H}]^{+}\right), 367.2380$; found, 367.2380 .

\section{Data for $4 \mathrm{~g}$}

Yield: $72 \%$, pale yellow solid, mp 90-92 ${ }^{\circ} \mathrm{C} ;[\alpha]_{\mathrm{D}}^{20}=-15(c$ $\left.2.7 \mathrm{mg} \mathrm{mL}{ }^{-1}, \mathrm{CHCl}_{3}\right)$; IR cm ${ }^{-1}(\mathrm{KBr}): 3002,2743,1628,1562$, 1507; ${ }^{1} \mathrm{H}$ NMR $\left(500 \mathrm{MHz}, \mathrm{CDCl}_{3}\right) \delta: 9.35(\mathrm{~s}, 1 \mathrm{H}), 7.08(\mathrm{~d}, J=$ $8.0 \mathrm{~Hz}, 2 \mathrm{H}), 6.89$ (d, $J=8.5 \mathrm{~Hz}, 2 \mathrm{H}), 3.95-3.99(\mathrm{~m}, 1 \mathrm{H}), 3.48$ $(\mathrm{dd}, J=13.0,4.5 \mathrm{~Hz}, 1 \mathrm{H}), 3.31(\mathrm{t}, J=13.0 \mathrm{~Hz}, 1 \mathrm{H}), 2.84-2.74$ (m, 2H), 2.35-2.50 (m, 2H), $2.30(\mathrm{~s}, 3 \mathrm{H}), 2.11(\mathrm{~s}, 1 \mathrm{H}), 1.89-2.04$ (m, 4H), 1.72-1.78 (m, 1H), 1.55-1.67 (m, 4H), 1.39-1.45 (m, $5 \mathrm{H})$; HRMS (ESI): calcd for $\mathrm{C}_{23} \mathrm{H}_{31} \mathrm{O}_{2} \mathrm{~N}_{2}\left([\mathrm{M}+\mathrm{H}]^{+}\right)$, 367.2380; found, 367.2379 .

\section{Data for $4 h$}

Yield: $70 \%$, pale yellow solid, $\mathrm{mp} 87-89{ }^{\circ} \mathrm{C} ;[\alpha]_{\mathrm{D}}^{20}=-18(c$ $\left.2.6 \mathrm{mg} \mathrm{mL}{ }^{-1}, \mathrm{CHCl}_{3}\right)$; IR cm ${ }^{-1}(\mathrm{KBr}): 3057,2762,1635,1579$, 1501; ${ }^{1} \mathrm{H}$ NMR $\left(500 \mathrm{MHz}, \mathrm{CDCl}_{3}\right) \delta: 9.40(\mathrm{~s}, 1 \mathrm{H}), 6.94(\mathrm{~d}, J=$ $9.0 \mathrm{~Hz}, 2 \mathrm{H}), 6.82(\mathrm{~d}, J=9.0 \mathrm{~Hz}, 2 \mathrm{H}), 3.93-3.97(\mathrm{~m}, 1 \mathrm{H}), 3.77(\mathrm{~s}$, $3 \mathrm{H}), 3.48(\mathrm{dd}, J=14.0,5.0 \mathrm{~Hz}, 1 \mathrm{H}), 3.31(\mathrm{t}, J=12.5 \mathrm{~Hz}, 1 \mathrm{H})$, 2.74-2.84 (m, 2H), 2.33-2.50 (m, 2H), $2.10(\mathrm{~s}, 1 \mathrm{H}), 1.91-2.03$ (m, 4H), 1.71-1.76 (m, 1H), 1.52-1.64 (m, 4H), 1.37-1.45 (m, $5 \mathrm{H})$; HRMS (ESI): calcd for $\mathrm{C}_{23} \mathrm{H}_{31} \mathrm{O}_{3} \mathrm{~N}_{2}\left([\mathrm{M}+\mathrm{H}]^{+}\right)$, 383.2329; found, 383.2326 .

\section{Data for $4 i$}

Yield: $65 \%$, white solid, $\mathrm{mp} 100-102{ }^{\circ} \mathrm{C} ;[\alpha]_{\mathrm{D}}^{20}=-30(c 2.6 \mathrm{mg}$ $\mathrm{mL}^{-1}, \mathrm{CHCl}_{3}$ ); IR cm ${ }^{-1}$ (KBr): 3055, 2774, 1628, 1562, $1505 ;{ }^{1} \mathrm{H}$ NMR (500 MHz, $\left.\mathrm{CDCl}_{3}\right) \delta: 9.45(\mathrm{~s}, 1 \mathrm{H}), 7.79(\mathrm{t}, J=8.0 \mathrm{~Hz}, 2 \mathrm{H})$, $7.71(\mathrm{~d}, J=8.5 \mathrm{~Hz}, 1 \mathrm{H}), 7.46(\mathrm{t}, J=8.0 \mathrm{~Hz}, 1 \mathrm{H}), 7.39(\mathrm{~d}, J=$ $7.5 \mathrm{~Hz}, 1 \mathrm{H}), 7.33(\mathrm{~s}, 1 \mathrm{H}), 7.26-7.28(\mathrm{~m}, 1 \mathrm{H}), 4.01-4.03(\mathrm{~m}, 1 \mathrm{H})$, $3.51(\mathrm{dd}, J=13.0,4.0 \mathrm{~Hz}, 1 \mathrm{H}), 3.34(\mathrm{t}, J=12.5 \mathrm{~Hz}, 1 \mathrm{H}), 2.73-2.84$ (m, 2H), 2.41-2.54 (m, 2H), 2.09-2.11 (m, 2H), 1.88-2.00 (m, $3 \mathrm{H}), 1.80-1.84(\mathrm{~m}, 1 \mathrm{H}), 1.58-1.71(\mathrm{~m}, 4 \mathrm{H}), 1.34-1.47(\mathrm{~m}, 5 \mathrm{H})$; HRMS (ESI): calcd for $\mathrm{C}_{26} \mathrm{H}_{31} \mathrm{O}_{2} \mathrm{~N}_{2}\left([\mathrm{M}+\mathrm{H}]^{+}\right), 403.2380$; found, 403.2377.

\section{Data for $4 \mathbf{j}$}

Yield: $58 \%$, white solid, $\mathrm{mp} 103-105{ }^{\circ} \mathrm{C} ;[\alpha]_{\mathrm{D}}^{20}=-11(c 2.8 \mathrm{mg}$ $\mathrm{mL}^{-1}, \mathrm{CHCl}_{3}$ ); IR cm ${ }^{-1}$ (KBr): 3057, 2757, 1635, 1595, 1576, 1507; ${ }^{1} \mathrm{H}$ NMR $\left(500 \mathrm{MHz}, \mathrm{CDCl}_{3}\right) \delta: 9.41(\mathrm{~s}, 1 \mathrm{H}), 8.28-8.30(\mathrm{~m}$, $1 \mathrm{H}), 7.85-7.87(\mathrm{~m}, 1 \mathrm{H}), 7.54-7.57(\mathrm{~m}, 3 \mathrm{H}), 7.32(\mathrm{t}, J=8.0 \mathrm{~Hz}$, $1 \mathrm{H}), 6.97(\mathrm{~d}, J=7.5 \mathrm{~Hz}, 1 \mathrm{H}), 4.03(\mathrm{~s}, 1 \mathrm{H}), 3.41$ (dd, $J=13.0$, $4.5 \mathrm{~Hz}, 1 \mathrm{H}), 3.32$ (t, $J=13.0 \mathrm{~Hz}, 1 \mathrm{H}), 2.82(\mathrm{~d}, J=10.5 \mathrm{~Hz}, 1 \mathrm{H})$, $2.72(\mathrm{~d}, J=11.5 \mathrm{~Hz}, 1 \mathrm{H}), 2.47$ (s, 2H), 1.87-2.13 (m, 6H), 1.58$1.75(\mathrm{~m}, 4 \mathrm{H}), 1.39-1.56(\mathrm{~m}, 5 \mathrm{H})$; HRMS (ESI): calcd for $\mathrm{C}_{26} \mathrm{H}_{31} \mathrm{O}_{2} \mathrm{~N}_{2}\left([\mathrm{M}+\mathrm{H}]^{+}\right)$, 403.2380; found, 403.2377.

\section{Data for $\mathbf{4 k}$}

Yield: $49 \%$, pale yellow solid, $\operatorname{mp} 162-164{ }^{\circ} \mathrm{C} ;[\alpha]_{\mathrm{D}}^{20}=32(c$ $3.0 \mathrm{mg} \mathrm{mL}{ }^{-1}, \mathrm{CHCl}_{3}$ ); IR cm ${ }^{-1}$ (KBr): 3111, 3029, 2771, 1675, 1650, 1613, 1588, 1553, 1513, 1494; ${ }^{1} \mathrm{H}$ NMR (500 MHz, $\mathrm{CDCl}_{3}$ ) $\delta: 8.21(\mathrm{~d}, J=8.5 \mathrm{~Hz}, 2 \mathrm{H}), 7.16(\mathrm{~d}, J=8.5 \mathrm{~Hz}, 2 \mathrm{H}), 6.60(\mathrm{~s}, 1 \mathrm{H})$, $4.46(\mathrm{dd}, J=12.5,3.5 \mathrm{~Hz}, 1 \mathrm{H}), 3.89-3.94(\mathrm{~m}, 1 \mathrm{H}), 3.09(\mathrm{t}, J=$ $13.0 \mathrm{~Hz}, 1 \mathrm{H}), 2.78-2.86(\mathrm{~m}, 2 \mathrm{H}), 2.27-2.41(\mathrm{~m}, 3 \mathrm{H}), 2.10(\mathrm{~s}, 1 \mathrm{H})$, 1.87-2.01 (m, 3H), 1.62-1.75 (m, 4H), 1.39-1.55 (m, 6H); HRMS (ESI): calcd for $\mathrm{C}_{22} \mathrm{H}_{28} \mathrm{O}_{4} \mathrm{~N}_{3}\left([\mathrm{M}+\mathrm{H}]^{+}\right)$, 398.2074; found, 398.2073.

\section{Data for 41}

Yield: $33 \%$, pale yellow solid, mp $152-154{ }^{\circ} \mathrm{C} ;[\alpha]_{\mathrm{D}}^{20}=18(\mathrm{c}$ $\left.3.2 \mathrm{mg} \mathrm{mL}{ }^{-1}, \mathrm{CHCl}_{3}\right)$; IR cm ${ }^{-1}(\mathrm{KBr}): 3070,3026,2774,1697$, 1672, 1596, 1505; ${ }^{1} \mathrm{H}$ NMR (500 MHz, $\mathrm{CDCl}_{3}$ ) $\delta: 9.92$ (s, 1H), 7.85 $(\mathrm{d}, J=8.5 \mathrm{~Hz}, 2 \mathrm{H}), 7.20$ (d, $J=8.5 \mathrm{~Hz}, 2 \mathrm{H}), 6.63(\mathrm{~s}, 1 \mathrm{H}), 4.48$ (dd, $J=12.5,3.0 \mathrm{~Hz}, 1 \mathrm{H}), 3.89-3.92(\mathrm{~m}, 1 \mathrm{H}), 3.08(\mathrm{t}, J=12.5 \mathrm{~Hz}, 1 \mathrm{H})$, 2.78-2.86 (m, 2H), 2.26-2.40 (m, 3H), 2.10 (s, 1H), 1.87-2.01 (m, $3 \mathrm{H})$, 1.63-1.74 (m, 4H), 1.42-1.55 (m, 6H); HRMS (ESI): calcd for $\mathrm{C}_{23} \mathrm{H}_{29} \mathrm{O}_{3} \mathrm{~N}_{2}\left([\mathrm{M}+\mathrm{H}]^{+}\right)$, 381.2173; found, 381.2172. 


\section{Biological assay}

Oral toxicity of 1 and 4a, d, e, g, i, k against Plutella xylostella. Thirty 3rd-instar larvae of $P$. xylostella were chosen as the test insects for each compound. Solutions of $\mathbf{1} ; \mathbf{4 a}, \mathbf{d}, \mathbf{e}, \mathbf{g}, \mathbf{i}, \mathbf{k}$; and toosendanin (a positive control) were prepared in acetone at $20 \mathrm{mg} \mathrm{mL}^{-1}$. The corresponding solution $(1 \mu \mathrm{L})$ was added to a fresh Brassica oleracea leaf disc $(0.5 \times 0.5 \mathrm{~cm})$, and dried. A fresh Brassica oleracea leaf disc was treated by acetone alone as the blank control group (CK). One piece of the above discs was offered to and consumed by each insect, which was raised in each well of 12- or 24-well culture plates for $48 \mathrm{~h}$ (temperature: $25 \pm 2{ }^{\circ} \mathrm{C}$; relative humidity $(\mathrm{RH}): 65-80 \%$; photoperiod: light/ dark $(\mathrm{L} / \mathrm{D})=16 / 8 \mathrm{~h})$. Their corrected mortality rate values were calculated as follows: corrected mortality rate $(\%)=(T-C)$ $\times 100 /(100 \%-C) ; C$ is the mortality rate of CK, and $T$ is the mortality rate of the treated $P$. xylostella.$^{23,24}$

Growth inhibitory activity of 1, 2, and 4a-l against Mythimna separata. Thirty early 3rd-instar larvae of $M$. separata were chosen as the test insects for each compound. Solutions of 1, 2, 4a-l, and toosendanin (a positive control) were prepared in acetone at $1 \mathrm{mg} \mathrm{mL} \mathrm{mL}^{-1}$. After dipping into the corresponding solution for $3 \mathrm{~s}$, wheat leaf discs $(1 \times 1 \mathrm{~cm})$ were taken out, and dried. Wheat leaf discs were treated by acetone alone as the blank control group (CK). Several of the above discs were added to each culture dish (ten insects per dish). Once the discs had been consumed, additional ones were added. After $48 \mathrm{~h}$, the rest of the compound-soaked discs were cleaned out, and untreated ones were added until the end of pupation (temperature: $25 \pm$ $2{ }^{\circ} \mathrm{C}$; RH: $65-80 \%$; photoperiod: $\mathrm{L} / \mathrm{D}=12 / 12 \mathrm{~h}$ ). Their corrected mortality rate values were calculated as follows: corrected mortality rate $(\%)=(T-C) \times 100 /(100 \%-C) ; C$ is the mortality rate of $\mathrm{CK}$, and $T$ is the mortality rate of the treated $M$. separata. $^{25,26}$

\section{Results and discussion}

\section{Synthesis}

As shown in Scheme 1, first, the key intermediate, 14-formyl-15chloromatrine (2), was smoothly synthesized by the VilsmeierHaack reaction of matrine (1) with DMF in the presence of $\mathrm{POCl}_{3}$. Then, 14-formyl-15-aryloxy/methoxymatrine derivatives (4a-e) were easily prepared by the reaction of 2 with corresponding phenols (3a-d) or methanol (3e) in the presence of $\mathrm{KOH}$ at $120{ }^{\circ} \mathrm{C}$ or under reflux..$^{27}$ However, when 2 reacted with $\mathbf{3 f}-\mathbf{j}$ under the above reaction conditions, it was very difficult to separate 14-formyl-15-aryloxymatrine derivatives (4f-j) from their by-products. It is noteworthy that when 2 reacted with $\mathbf{3 f}-\mathbf{j}$ under microwave irradiation at $120{ }^{\circ} \mathrm{C}$ for $20 \mathrm{~min}$, compounds $\mathbf{4 f}-\mathbf{j}$ were smoothly obtained. Interestingly, when 2 reacted with $3 \mathbf{k}, \mathbf{l}$ in the presence of $\mathrm{KOH}$ at $120{ }^{\circ} \mathrm{C}$, only 14-aryloxymethylidenylmatrines $(\mathbf{4 k}, \mathbf{l})$ were produced. Their structures were well determined by melting points, optical rotation, IR, ${ }^{1} \mathrm{H}$ NMR, and HRMS. In particular, three-dimensional structures of compounds $\mathbf{2}, \mathbf{4 d}, \mathbf{4 g}$ and $\mathbf{4 k}$ were determined by X-ray crystallography (Fig. 2-5). Crystallographic data (excluding structure factors) of $\mathbf{2}, \mathbf{4 d}, \mathbf{4} \mathbf{g}$ and $\mathbf{4 k}$ were deposited at the Cambridge Crystallographic Data Centre (CCDC) with deposition numbers of 1522361, 1524882, 1524886 and 1524887, respectively. Assignment of the chemical structure for $\mathbf{4 1}$ was based on the

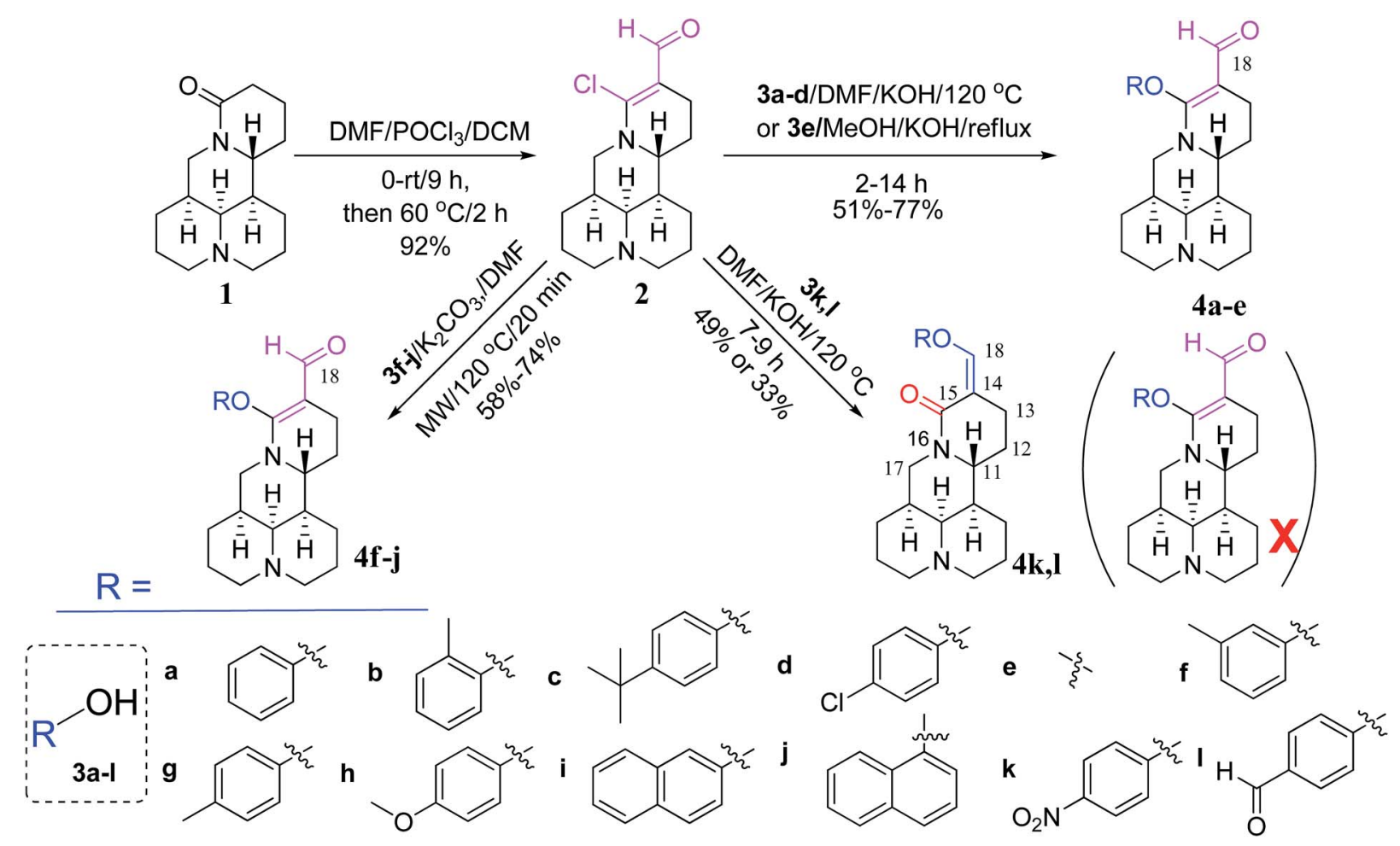

Scheme 1 Synthesis of 14-formyl-15-aryloxy/methoxymatrines (4a-j) and 14-aryloxymethylidenylmatrines (4k, l) from matrine. 


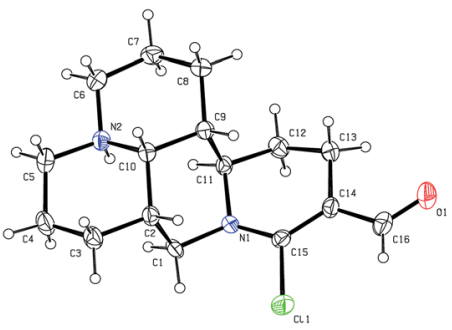

Fig. 2 X-ray crystal structure of compound 2.

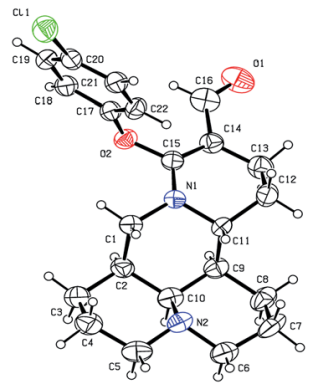

Fig. 3 X-ray crystal structure of compound 4 d.

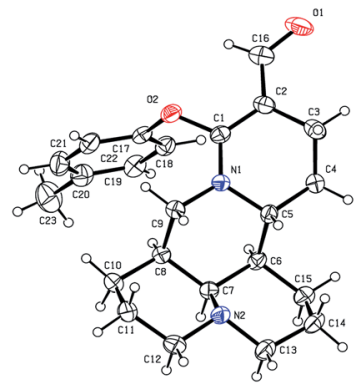

Fig. 4 X-ray crystal structure of compound $4 \mathrm{~g}$.

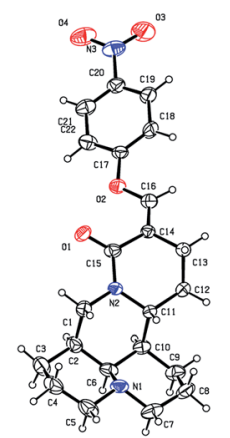

Fig. 5 X-ray crystal structure of compound $4 \mathrm{k}$.

chemical shift of H-18. As shown in Fig. 6, the chemical shifts of $\mathrm{H}-18$ of $2, \mathbf{4 d}$, and $\mathbf{4 g}$ were at 9.67, 9.29, and 9.35 ppm, respectively. Whereas the chemical shift of $\mathrm{H}-18$ of $4 \mathbf{k}$ was at $6.60 \mathrm{ppm}$. Here the chemical shift of $\mathrm{H}-18$ of 41 was at $6.63 \mathrm{ppm}$, so the steric structure for $\mathbf{4 l}$ was the same as that of $\mathbf{4 k}$.

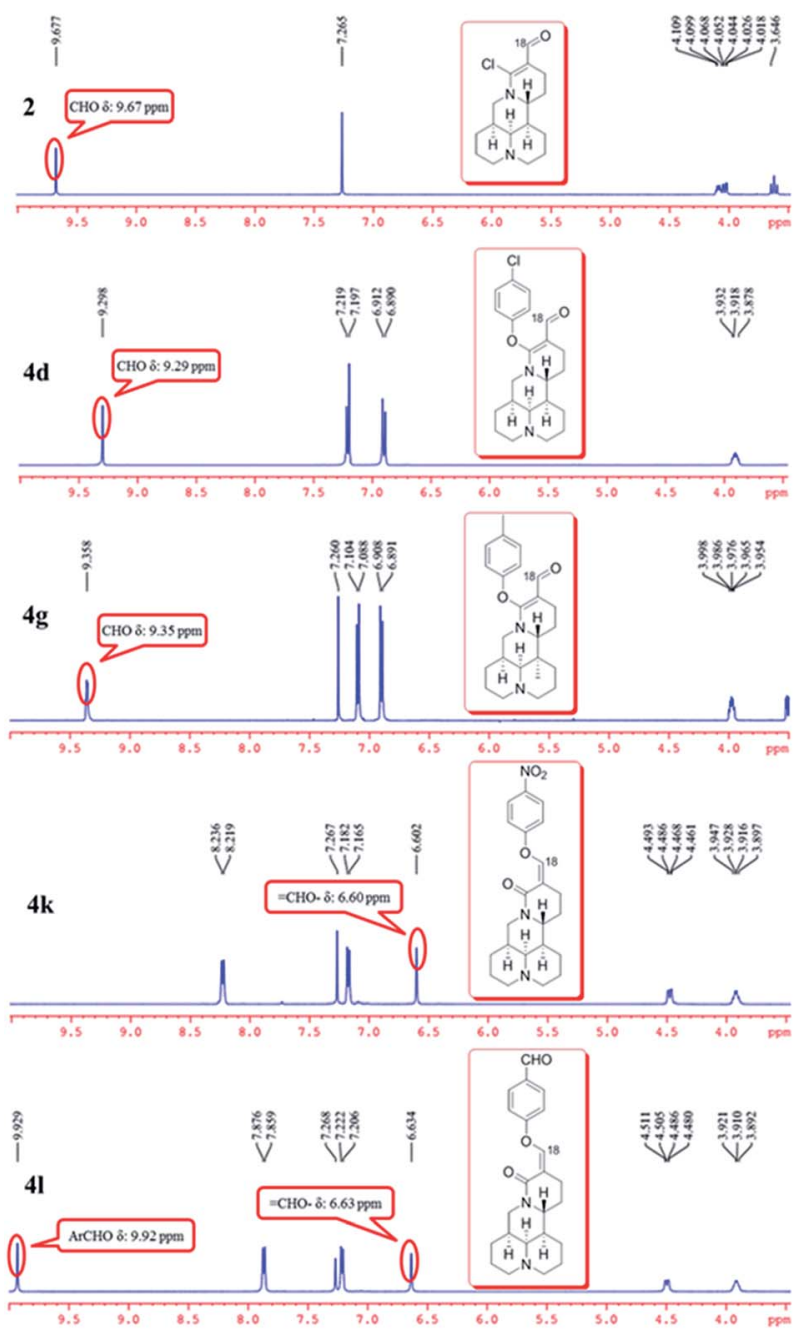

Fig. 6 Comparison of partial ${ }^{1} \mathrm{H}$ NMR spectra of compounds $2,4 \mathrm{~d}, 4 \mathrm{~g}$, $4 \mathrm{k}$, and $4 \mathrm{l}$.

The possible mechanism for $\mathbf{3 a - 1}$ reacting with 2 is described in Fig. 7. Compounds $\mathbf{3 a - j}$ reacted with 2 via the intermediates $\mathbf{5 a}-\mathbf{j}$ to afford 14-formyl-15-aryloxy/methoxymatrine derivatives $(\mathbf{4 a}-\mathbf{j})$ by the 1,4-addition-elimination reaction (eqn (1)). However, for compounds $\mathbf{3 k}$, $\mathbf{l}$, first, the hydroxyl ion reacted with 2 via the intermediate 6 to afford 14-formyl-15-hydroxylmatrine (7) by the 1,4-addition-elimination reaction. Then, compound 7 was converted into intermediate 8 . Finally, compound 8 reacted with $3 \mathbf{k}, \mathbf{l}$, followed by removal of a molecule of water to give 14aryloxymethylidenylmatrines (4k, l) (eqn (2)).

\section{Insecticidal activity}

The oral toxicity of compounds $\mathbf{1}$ and $\mathbf{4 a}, \mathbf{d}, \mathbf{e}, \mathbf{g}, \mathbf{i}, \mathbf{k}$ against $P$. xylostella treated at $20 \mu \mathrm{g} /$ larvae is described in Table 1 . The mortality rates after $48 \mathrm{~h}$ of $\mathbf{1}, \mathbf{4 a}, \mathbf{4 d}, \mathbf{4 e}, \mathbf{4 g}, \mathbf{4 i}$, and $4 \mathbf{k}$ were $28.5 \%, 32.1 \%$, 35.7\%, 35.7\%, 32.1\%, 39.3\%, and $42.8 \%$, respectively. Among them, compounds 4d, 4e, 4i, and $4 \mathbf{k}$ exhibited potent oral toxicity when compared with toosendanin. The growth inhibitory activity of compounds $\mathbf{1}, \mathbf{2}$, and $\mathbf{4 a - 1}$ against $M$. separata was tested at $1 \mathrm{mg} \mathrm{mL}^{-1}$. As shown in Table 2, 


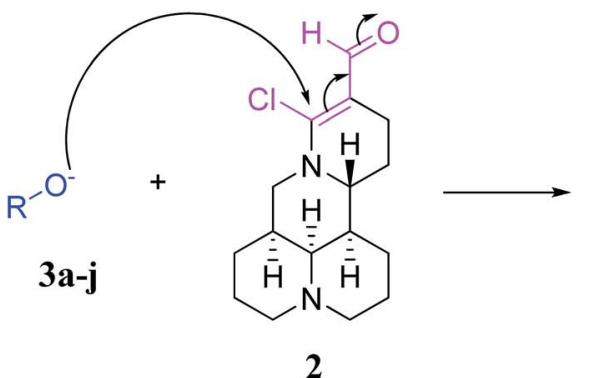

2

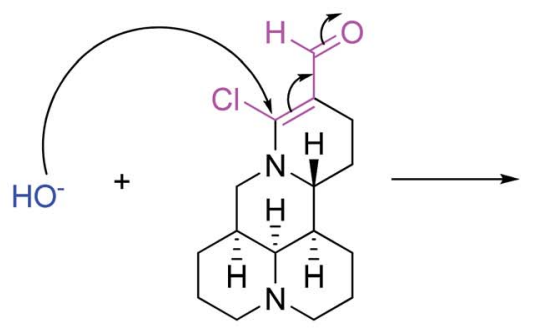

2

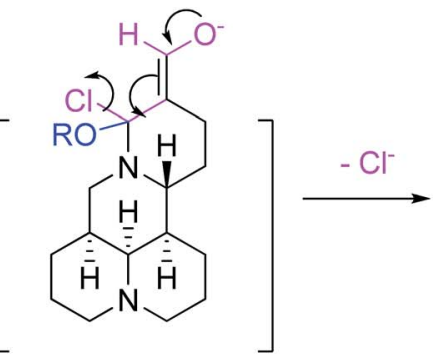

$\mathbf{5 a - j}$<smiles>[R9]C1=C(C=O)CC[C@@]2(C)[C@H]3CCCN4CCC[C@@H](CN12)[C@@H]34</smiles>

4a-j

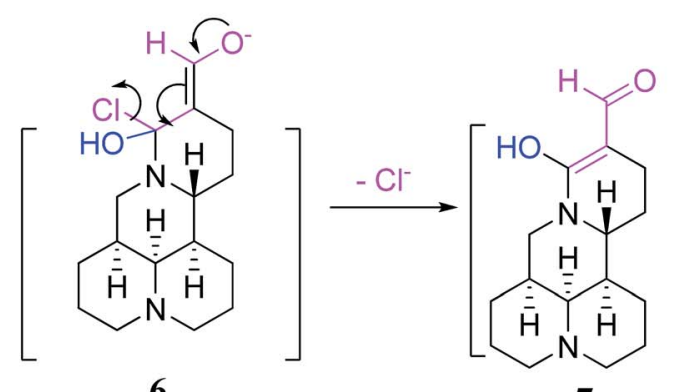

6

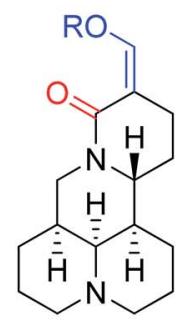

$4 \mathrm{k}, 1$

Fig. 7 Possible mechanism for $3 a-1$ reacting with 2.

compounds $\mathbf{4 i}, \mathbf{4} \mathbf{j}$ and $\mathbf{4 k}$ showed higher insecticidal activity than toosendanin. For example, the final mortality rates (FMRs) of $\mathbf{4 i}$, $4 \mathbf{j}$ and $4 \mathbf{k}$ were $62.1 \%, 58.6 \%$, and $55.2 \%$, respectively; whereas the FMR of 1 was only $24.2 \%$. In particular, compound $4 \mathbf{i}$ exhibited the most potent insecticidal activity. The symptoms for

Table 1 Oral toxicity of compounds 1 and $4 \mathrm{a}, \mathrm{d}, \mathrm{e}, \mathrm{g}, \mathrm{i}, \mathrm{k}$ against $P$ xylostella treated at $20 \mu \mathrm{g} /$ Larvae

\begin{tabular}{llc}
\hline & \multicolumn{2}{l}{ Corrected mortality rate $($ mean $\pm \mathrm{SD}, \%)$} \\
\cline { 2 - 3 } Compound & $24 \mathrm{~h}$ & $48 \mathrm{~h}$ \\
\hline $\mathbf{1}$ & $10.4 \pm 0$ & $28.5 \pm 3.3 \mathrm{c}^{a}$ \\
$\mathbf{4 a}$ & $13.8 \pm 3.3$ & $32.1 \pm 3.3 \mathrm{bc}$ \\
$\mathbf{4 d}$ & $10.4 \pm 3.3$ & $35.7 \pm 5.8 \mathrm{abc}$ \\
$\mathbf{4 e}$ & $13.8 \pm 3.3$ & $35.7 \pm 0 \mathrm{abc}$ \\
$\mathbf{4 g}$ & $10.4 \pm 3.3$ & $32.1 \pm 6.7 \mathrm{bc}$ \\
$\mathbf{4 i}$ & $10.4 \pm 3.3$ & $39.3 \pm 3.3 \mathrm{abc}$ \\
$\mathbf{4 k}$ & $17.3 \pm 0$ & $42.8 \pm 3.3 \mathrm{ab}$ \\
Toosendanin & $0 \pm 3.3$ & $46.4 \pm 0 \mathrm{a}$
\end{tabular}

${ }^{a}$ Multiple range test using Duncan's test $(p<0.05)$. The same letters denote treatments not significantly different from each other.
Table 2 Growth inhibitory activity of compounds 1, 2, and 4a-1 against $M$. separata on leaves treated with a concentration of $1 \mathrm{mg}$ $\mathrm{mL}^{-1}$

\begin{tabular}{lcll}
\hline & \multicolumn{3}{c}{ Corrected mortality rate $($ mean $\pm \mathrm{SD}, \%)$} \\
\cline { 2 - 4 } Compound & 10 days & 25 days & 35 days \\
\hline $\mathbf{1}$ & $3.3 \pm 3.3$ & $16.7 \pm 3.3$ & $24.2 \pm 3.3 \mathrm{e}^{a}$ \\
$\mathbf{2}$ & $6.7 \pm 3.3$ & $23.3 \pm 3.3$ & $31.1 \pm 6.7 \mathrm{de}$ \\
$\mathbf{4 a}$ & $10.0 \pm 5.8$ & $26.7 \pm 6.7$ & $41.4 \pm 3.3 \mathrm{~cd}$ \\
$\mathbf{4 b}$ & $6.7 \pm 6.7$ & $23.3 \pm 6.7$ & $38.0 \pm 5.8 \mathrm{~cd}$ \\
$\mathbf{4 c}$ & $10.0 \pm 5.8$ & $23.3 \pm 3.3$ & $31.1 \pm 3.3 \mathrm{de}$ \\
$\mathbf{4 d}$ & $13.3 \pm 6.7$ & $30.0 \pm 5.8$ & $48.3 \pm 0 \mathrm{bc}$ \\
$\mathbf{4 e}$ & $20.0 \pm 0$ & $33.3 \pm 3.3$ & $48.3 \pm 5.8 \mathrm{bc}$ \\
$\mathbf{4 f}$ & $13.3 \pm 6.7$ & $33.3 \pm 3.3$ & $41.4 \pm 3.3 \mathrm{~cd}$ \\
$\mathbf{4 g}$ & $10.0 \pm 0$ & $26.7 \pm 3.3$ & $38.0 \pm 5.8 \mathrm{~cd}$ \\
$\mathbf{4 h}$ & $10.0 \pm 5.8$ & $30.0 \pm 5.8$ & $41.4 \pm 3.3 \mathrm{~cd}$ \\
$\mathbf{4 i}$ & $13.3 \pm 3.3$ & $40.0 \pm 0$ & $62.1 \pm 3.3 \mathrm{a}$ \\
$\mathbf{4 j}$ & $13.3 \pm 3.3$ & $36.7 \pm 3.3$ & $58.6 \pm 0 \mathrm{ab}$ \\
$\mathbf{4 k}$ & $20.0 \pm 0$ & $33.3 \pm 6.7$ & $55.2 \pm 3.3 \mathrm{ab}$ \\
$\mathbf{4 1}$ & $6.7 \pm 3.3$ & $23.3 \pm 3.3$ & $38.0 \pm 5.8 \mathrm{~cd}$ \\
Toosendanin & $16.7 \pm 6.7$ & $33.3 \pm 3.3$ & $48.3 \pm 0 \mathrm{bc}$
\end{tabular}

${ }^{a}$ Multiple range test using Duncan's test $(p<0.05)$. The same letters denote treatments not significantly different from each other. 
the treated M. separata during the larval, pupation and adult periods were observed. Fig. 8 shows the dead larvae with thin and wrinkled bodies at the larval stage; Fig. 9 shows some malformed and dead pupae during the pupation stage; Fig. 10 shows some malformed moths during the adult emergence stage. This demonstrates that matrine derivatives probably affected the insect molting hormone. On the other hand, the times for three growth periods of $M$. separata treated with 1, 2, and $\mathbf{4 a - 1}$ are shown in Fig. 11. This suggests that the times from the larvae to the adult in the treated groups were generally prolonged (33-35 days $v s$. 32 days for CK). Finally, the percentages of FMRs at three different growth stages of compounds $4 \mathbf{d}, 4 \mathbf{e}, 4 \mathbf{i}, 4 \mathbf{j}, \mathbf{4 k}$ and toosendanin were investigated, as displayed in Fig. 12. More than/ equal to half of FMRs for compounds $4 \mathbf{d}, \mathbf{4 e}, 4 \mathbf{i}, 4 \mathbf{j}, 4 \mathbf{k}$ and

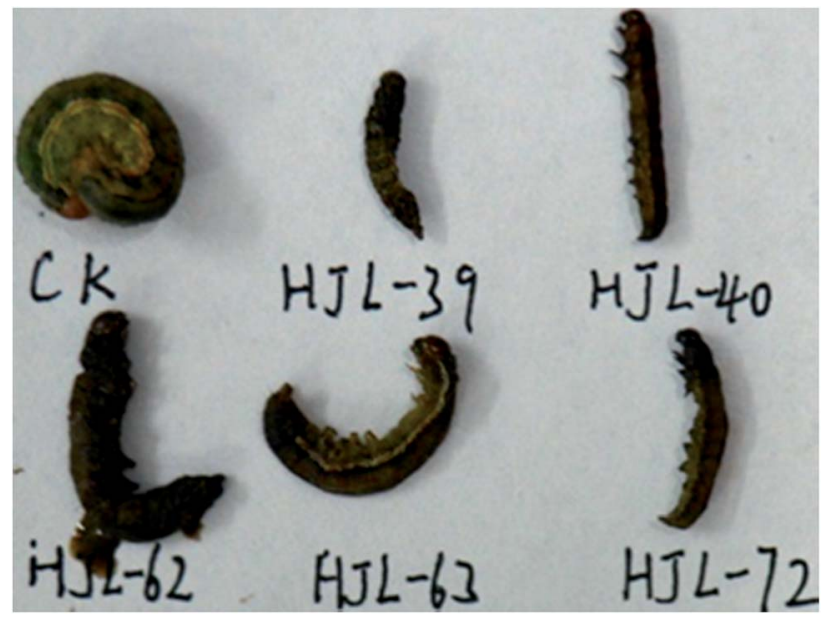

Fig. 8 The representative abnormal larvae pictures of $4 \mathrm{~d}(\mathrm{HJL}-39), 4 \mathrm{k}$ (HJL-40), 4i (HJL-62), 4j (HJL-63), and 4e (HJL-72) during the larval period (CK: blank control group).

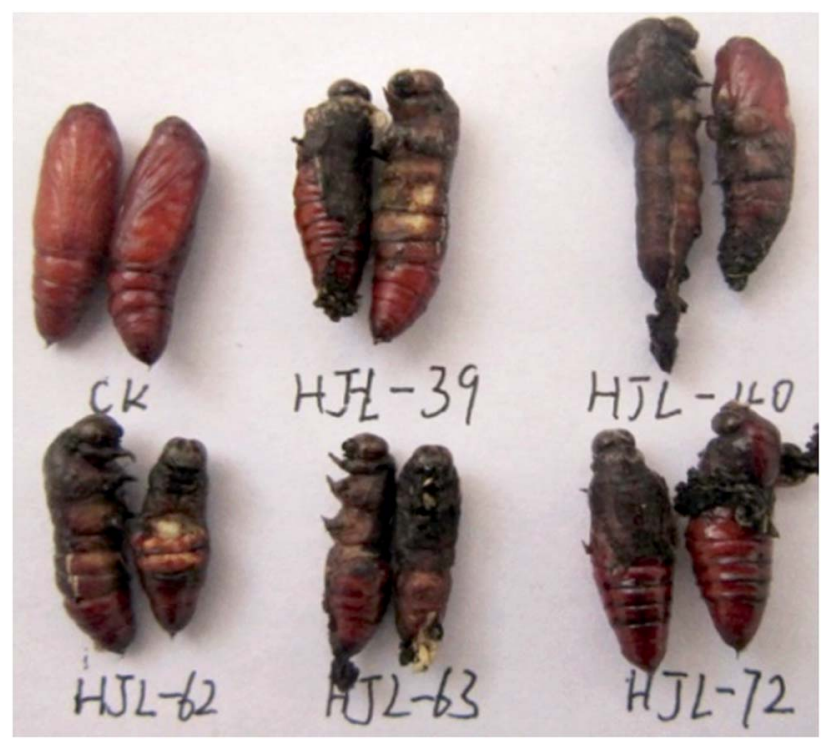

Fig. 9 The representative malformed pupae pictures of $4 d(H J L-39)$, $4 \mathrm{k}(\mathrm{HJL}-40), 4 \mathrm{i}(\mathrm{HJL}-62), 4 \mathrm{j}(\mathrm{HJL}-63)$, and $4 \mathrm{e}(\mathrm{HJL}-72)$ during the pupation period (CK: blank control group). toosendanin were at the larval stage. This result was the same with those of esters of fraxinellone C4/10-oxime. ${ }^{24}$

Additionally, some interesting results of structure-activity relationships of the tested compounds were also obtained. When the chlorine atom of 2 was substituted by other aryloxy/ methoxy groups such as 4-chlorophenyloxy, methoxy, 2-naphthyloxy, and 1-naphthyloxy, the corresponding products $4 \mathbf{d}$ (FMR: 48.3\%), 4e (FMR: 48.3\%), 4i (FMR: 62.1\%), and 4j (FMR: 58.6\%) showed more potent insecticidal activity than 1 (FMR: 24.2\%) and 2 (FMR: 31.1\%). Interestingly, one 14-formyl-15alkyloxymatrine, 4e (containing a methoxy group), exhibited promising insecticidal activity, so in the future other alkyloxy groups could be considered for introduction at the C-15 position of 2. In general, the introduction of an electronwithdrawing group on the phenyl ring of 4a (FMR: 41.4\%)

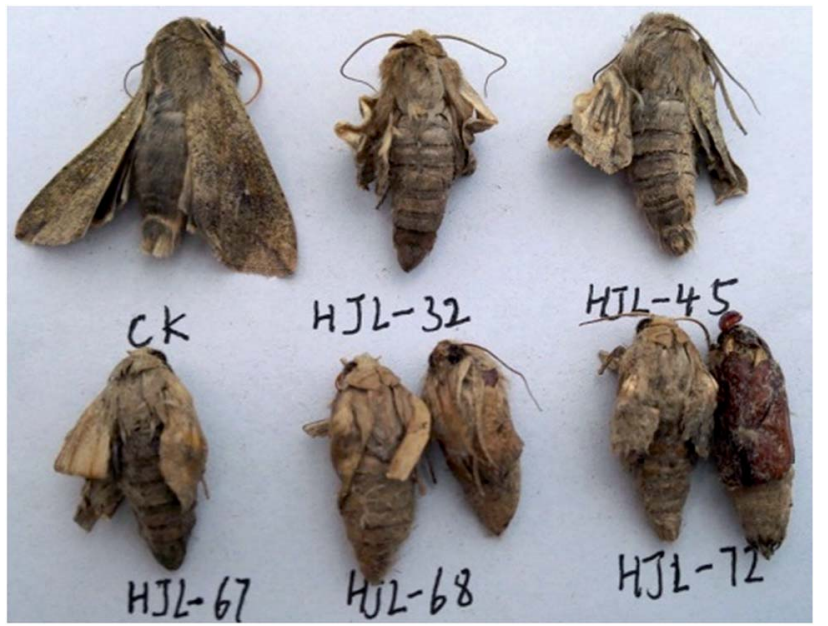

Fig. 10 The representative malformed moth pictures of $4 a(H J L-32)$, 4b (HJL-45), 4f (HJL-67), 4h (HJL-68), and 4e (HJL-72) during the emergence period (CK: blank control group).

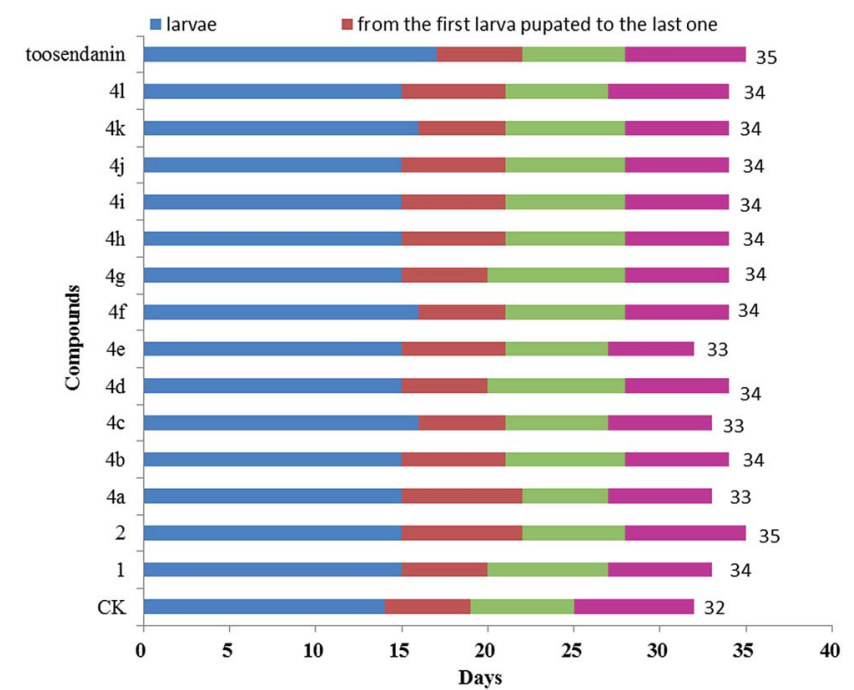

Fig. 11 Times for different developmental stages of $M$. separata treated with 1,2 , and $4 a-l$. 


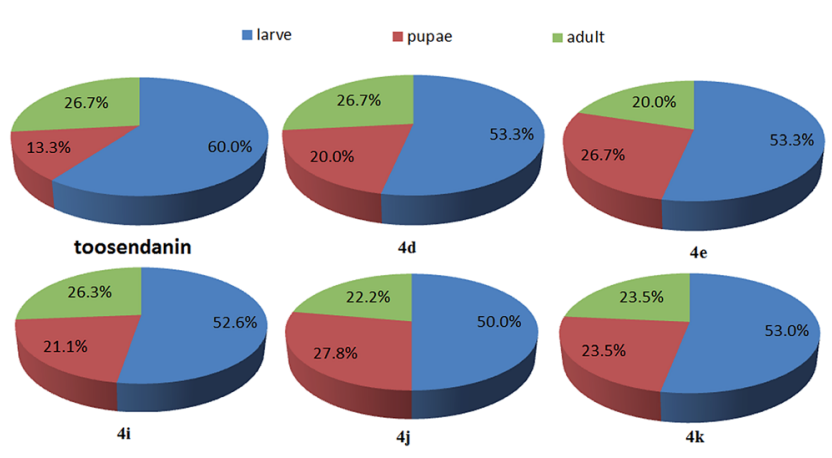

Fig. 12 The percentages of FMRs at three different growth stages of compounds $4 \mathrm{~d}, 4 \mathrm{e}, 4 \mathrm{i}, 4 \mathrm{j}, 4 \mathrm{k}$ and toosendanin.

could result in a more potent compound. For example, the FMR of 4d (containing 4-chlorophenyl) was 48.3\%; whereas the FMRs of $\mathbf{4 b}$ (containing 2-methylphenyl), 4c (containing 4-t-butylphenyl), 4f (containing 3-methylphenyl), 4g (containing 4methylphenyl), and $\mathbf{4 h}$ (containing 4-methoxyphenyl), were $38.0 \%, 31.1 \%, 41.4 \%, 38.0 \%$, and $41.4 \%$, respectively. The introduction of a polycyclic aromatic hydrocarbon oxy at the C15 position of 2 led to more potent products $4 \mathbf{i}$ and $\mathbf{4 j}$. For example, the FMR of $\mathbf{4 a}$ was $41.4 \%$; whereas the FMRs of $\mathbf{4 i}$ and $4 \mathbf{j}$ were $62.1 \%$, and $58.6 \%$, respectively.

\section{Conclusions}

In summary, we prepared a series of 14-formyl-15-aryloxy/ methoxymatrine and 14-aryloxymethylidenylmatrine derivatives as pesticidal agents by structural modification of matrine. A possible reaction mechanism for 3a-1 reacting with 2 was also proposed. Their pesticidal activities were evaluated against two crop-threatening agricultural insect pests, $M$. separata and $P$. xylostella. In particular, compounds $4 \mathbf{i}$ and $4 \mathbf{k}$ exhibited more promising pesticidal activities than matrine against $M$. separata and $P$. xylostella. This demonstrated that the introduction of an electron-withdrawing group on the phenyl ring of 14-formyl-15phenyloxymatrine, and the introduction of an alkyloxy or polycyclic aromatic hydrocarbon oxy at the C-15 position of 14 -formyl15-chloromatrine could result in more potent compounds. This will lay the foundations for further structural modification and application of matrines as biorenewable pesticidal agents for agriculture.

\section{Acknowledgements}

The present research was partly supported by National Natural Science Foundation of China (No. 31672071), and Special Funds of Central Colleges Basic Scientific Research Operating Expenses (No. 2452015096) to H. X.

\section{Notes and references}

1 X. F. Jiang, L. Z. Luo, L. Zhang, T. W. Sappington and Y. Hu, Environ. Entomol., 2011, 40, 516-533.

2 J. Y. Sun, P. Liang and X. W. Gao, Pest Manage. Sci., 2012, 68, 285-289.
3 J. Zeng, Y. Jiang and J. Liu, Plant Prot., 2013, 39, 117-121.

4 H. Wei, J. Liu, B. Li, Z. X. Zhan, Y. X. Chen, H. J. Tian, S. Lin and X. J. Gu, Crop Prot., 2015, 76, 68-74.

5 D. G. Heckel, Science, 2012, 337, 1612-1614.

6 K. Etebari, M. J. Furlong and S. Asgari, Sci. Rep., 2015, 5, 14642. 7 J. K. Kim, S. R. Choi, J. Lee, S. Y. Park, S. Y. Song, J. Na, S. W. Kim, S. J. Kim, I. S. Nou and Y. H. Lee, J. Agric. Food Chem., 2013, 61, 11222-11230.

8 Q. Fu, Q. Fang, B. L. Feng, S. J. Sun, W. Du, A. Enijian, A. P. Xiao and C. Chang, Journal of Chromatography B, 2011, 879, 894-900.

9 Z. J. Wu, D. M. Sun, D. M. Fang, J. Z. Chen, P. Cheng and G. L. Zhang, Int. J. Mass Spectrom., 2013, 341-342, 28-33.

10 Y. Liu, Y. Xu, W. D. Ji, X. Y. Li, B. Sun, Q. G. Gao and C. Q. Su, Tumor Biol., 2014, 35, 5111-5119.

11 B. Zhang, Z. Y. Liu, Y. Y. Li, Y. Luo, M. L. Liu, H. Y. Dong, Y. X. Wang, Y. Liu, P. T. Zhao, F. G. Jin and Z. C. Li, Eur. J. Pharm. Sci., 2011, 44, 573-579.

12 H. G. Hu, S. Z. Wang, C. M. Zhang, L. Wang, L. Ding, J. P. Zhang and Q. Y. Wu, Bioorg. Med. Chem. Lett., 2010, 20, 7537-7539.

13 L. M. Gao, Y. X. Han, Y. P. Wang, Y. H. Li, Y. Q. Shan, X. Li, Z. G. Peng, C. W. Bi, T. A. Zhang, N. N. Du, J. D. Jiang and D. Q. Song, J. Med. Chem., 2011, 54, 869-876.

14 L. Q. He, H. X. Gu, D. K. Yin, Y. H. Zhang and X. S. Wang, Chemical Journal of Chinese Universities, 2010, 31, 1541-1547.

15 L. S. Wang, Y. J. You, S. Q. Wang, X. Liu, B. M. Liu, J. N. Wang, X. Lin, M. S. Chen, G. Liang and H. Yang, Bioorg. Med. Chem. Lett., 2012, 22, 4100-4102.

16 J. L. Huang and H. Xu, Curr. Top. Med. Chem., 2016, 16, 33653378.

17 Z. Z. Odimar, D. P. R. Leandro, F. A. Thiago, S. S. Monica, P. B. Gabriela, T. Y. Pedro and D. V. Jose, Crop Prot., 2015, 67, 160-167.

18 B. Z. Zhong, C. J. Lu, X. D. Sun, W. Q. Qin and Z. Q. Peng, Agrochemicals, 2010, 49, 924-926.

19 J. Yuan, L. Z. Lu, B. Cong, Z. J. Zhang and F. Y. Wang, Chin. J. Pestic., 2016, 16, 3365-3378.

20 M. Garrido, M. Cabeza, F. Cortes, J. Gutierrez and E. Bratoeff, Eur. J. Med. Chem., 2013, 68, 301-311.

21 A. Sanchez-Marquez, Y. Arellano, E. Bratoeff, Y. Heuze, K. Cordova, G. Nieves, J. Soriano and M. Cabeza, J. Enzyme Inhib. Med. Chem., 2016, 31, 1170-1176.

22 E. Bratoeff, M. Garrido, T. Ramirez-Apan, Y. Heuze, A. Sanchez, J. Soriano and M. Cabeza, Bioorg. Med. Chem., 2014, 22, 6233-6241.

23 M. Lv, W. J. Wu and H. X. Liu, Pestic. Biochem. Physiol., 2008, 90, 114-118.

24 Q. Li, X. B. Huang, S. C. Li, J. C. Ma, M. Lv and H. Xu, J. Agric. Food Chem., 2016, 64, 5472-5478.

25 R. Wang, X. Y. Zhi, J. Li and H. Xu, J. Agric. Food Chem., 2015, 63, 6668-6674.

26 X. Yu, D. Shi, X. Zhi, Q. Li, X. Yao and H. Xu, RSC Adv., 2015, 5, 31700-31707.

27 A. L. LaFrate, J. R. Gunther, K. E. Carlson and J. A. Katzenellenbogen, Bioorg. Med. Chem., 2008, 16, 10075-10084. 